\title{
Critical submergence for isolated and dual rectangular intakes
}

\author{
KEREM TAŞTAN \\ Department of Civil Engineering, Faculty of Engineering, Gazi University, 06570 Maltepe, Ankara, Turkey \\ e-mail: ktastan@gazi.edu.tr
}

MS received 15 July 2015; revised 2 October 2015; accepted 9 October 2015

\begin{abstract}
This study examined critical submergence for isolated and dual rectangular intakes. It is shown that the critical submergence for an isolated intake can be predicted by disregarding whole boundary blockages on the complete imaginary critical sink surface that is the combination of imaginary complete critical cylindrical and hemi-spherical sink surfaces. It is proposed that this theory can be applied to the rectangular intakes located in general geometrical and flow conditions (i.e., intake in still water, circulation imposed flow, non-developed cross-flow, multiple intakes, etc.) and that it does not require computation of blockages caused from flow boundaries. The concept of complete sink surface (disregarding whole boundary blockages) developed for an isolated intake was also applied to dual rectangular intakes. The agreement between available test data and theoretical results was found to be satisfactory.
\end{abstract}

Keywords. Air-entrainment; boundary; critical submergence; intake; vortex.

\section{Introduction}

Air-entrainment resulting from an air-core free surface vortex is a frequently encountered problem at intake structures. If the submergence of the intake, $S$, is below a certain value, $S_{c}$ (critical submergence), air is entrained to the intake by the air-core vortex (figure 1a). There are several analytical and experimental studies relating to free surface vortices occurring at pipe intakes. Borghei and Kabiri-Samani [1], Naderi et al [2], Trivellato [3], Mahyari et al [4] and Wang et al [5] investigated efficiencies of various anti-vortex devices. Suerich-Gulick et al [6], Taştan and Yıldırım [7, 8] studied the scale effects (or effects of Froude, Reynolds and Weber numbers) on air-entraining vortices. Sarkardeh et al [9] and Yang et al [10] researched the effects of head wall and intake-entrance profiles on air-entrainment to pipe intakes. Yıldırım and Kocabaş [11] obtained the critical submergence of a pipe intake located in a cross-flow by potential flow solution. Carriveau et al [12], Jiming et al [13] and Odgaard [14] examined velocity profiles and critical submergence for pipe intakes. Rectangular intakes are often used in reservoirs and selective withdrawal structures. Studies relating to critical submergence of rectangular intakes include Ylldırım [15], Yıldırım et al [16] and Eroğlu and Bahadırlı [17]

These previous efforts have attempted to find the critical submergence by potential flow solution and considered the "blockage effects" of the impervious solid boundaries. The loss in intake discharge due to a solid boundary is called the "blockage effect" and the computation of the blockages of the flow boundaries is generally laborious.
Here, unlike in previous studies, the critical submergence for a rectangular intake(s) is predicted by disregarding whole boundary blockages. Therefore, the resulting method is much more practical and it can be applied for the rectangular intakes located in general geometrical and flow conditions. The comparison of the theoretical results and the available test data reveals that the agreement is good.

\section{Case of an isolated rectangular intake}

Yıldırım [15] and Yıldırım et al [16, 18] indicated the followings for an intake.

(i) Flow towards to a pipe intake (circular crosssection) may approximately be considered as a point sink and the critical submergence may be predicted by using an imaginary complete critical spherical sink surface.

(ii) Similar to a pipe intake flow towards a square intake may be approximated as a point sink [spherical sink surface (SSS)].

(iii) Flow towards a rectangular intake admitting flow only from its long sides may be approximated as a line sink [cylindrical sink surface (CSS)].

Consider an isolated rectangular intake in critical conditions as shown in figure $1 \mathrm{~b}$. In figure $1 \mathrm{~b}, \mathrm{C}, \mathrm{M}_{1}$, and $\mathrm{M}_{2}$ are the center points of critical sink surfaces; $a$ and $b$ are the dimensions of the rectangular intake cross-section; and $c$ is the clearance of the intake to the canal bottom. A 


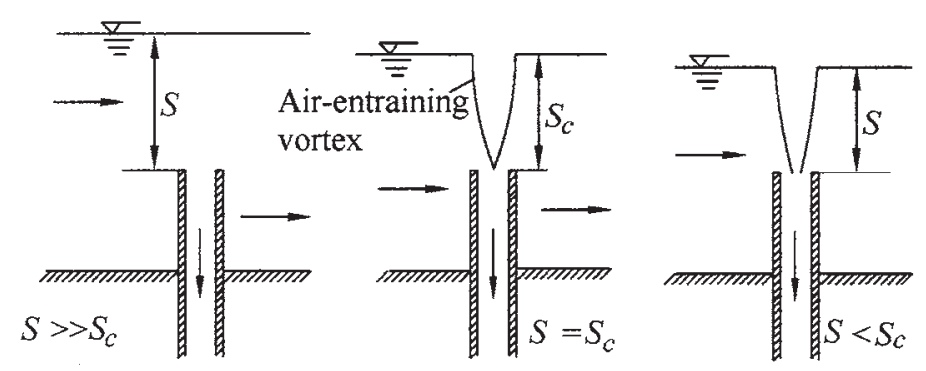

(a)

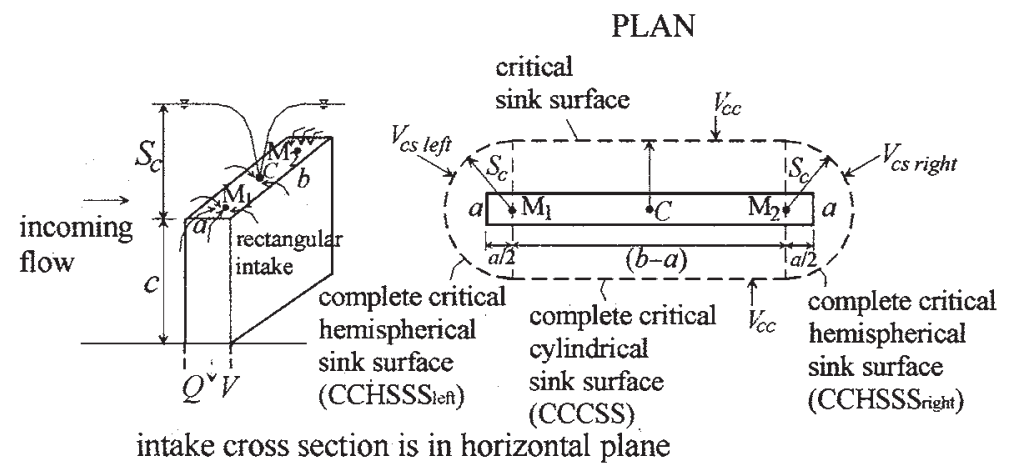

(b)

Figure 1. (a) Air-entrainment to an intake and (b) critical sink surface for an isolated rectangular intake [16].

rectangular intake may be split up into two main parts, as described in the following:

(a) The flow of half-square cross-sections having the dimensions of $a \times a / 2$ at both ends of the rectangular intake may be considered as hemispherical sink (HSS) flows. Taştan and Yıldırım [8] have proven that the critical submergence can be found by disregarding whole boundary blockages for a pipe intake. Herein, it is also reasonable to disregard whole boundary blockages and consider imaginary complete hemispherical sink surfaces at both ends (CHSSS left $_{\text {and }}$ CHSSS $\left.{ }_{\text {right }}\right)$. In these regions, under critical intake conditions, the CHSSS(s) are referred to as imaginary complete critical hemispherical sink surfaces CCHSSS $_{\text {left }}$ and CCHSSS $\left._{\text {right }}\right)$ that are tangent to water surface. The radii of CCHSSS(s) are equal to $S_{c}$ (figure $1 \mathrm{~b}$ ). The surface areas of CCHSSS(s) in figure $1 \mathrm{~b}$ are $A_{c s}$ left $=$ $A_{c s \text { right }}=2 \pi S_{c}^{2}$ and radial velocities at the CCHSSS left $_{\text {le }}$ and CCHSSS $_{\text {right }}$ are $V_{c s ~ l e f t}$, and $V_{c s}$ right, respectively.

(b) The flow towards the mid-section of the rectangular intake with a length of $(b-a)$ may be approximated as an imaginary complete cylindrical sink flow. Based on the same reason stated earlier, one can disregard whole boundary blockages and consider an imaginary complete cylindrical sink surface (CCSS). In this region, under critical intake conditions, the CCSS is referred to as an imaginary complete critical cylindrical sink surface (CCCSS) that is tangent to the water surface. The radius of CCCSS is equal to $S_{c}$ (figure 1b). The surface area of CCCSS is $A_{c c}=2 \pi S_{c}(b-a)$. The velocity at the CCCSS is $V_{c c}$.

In conclusion, critical sink surface of a rectangular intake is the combination of an imaginary complete critical cylindrical sink surface (CCCSS) with two imaginary complete critical hemispherical sink surfaces CCHSSS $_{\text {left }}$ and CCHSSS $_{\text {right }}$ ) (figure 1b).

For an isolated rectangular intake, $S_{c}$ may be obtained by means of any one of the following Eqs. (1a, 1b), (2), (3) and (4) (figure 1b).

Discharge through CCHSSS left, $_{\text {, }}$

$$
Q_{c s \text { left }}=A_{c s \text { left }} V_{c s \text { left }}=\left(a^{2} / 2\right) V
$$

Discharge through $\mathrm{CCHSSS}_{\text {right }}$,

$$
Q_{c s \text { right }}=A_{c s \text { right }} V_{c s \text { right }}=\left(a^{2} / 2\right) V .
$$

Discharge through two CCHSSS(s),

$$
Q_{c s}=Q_{c s \text { left }}+Q_{c s \text { right }}=A_{c s} V_{c s}=2\left(a^{2} / 2\right) V
$$

Discharge through CCCSS,

$$
Q_{c c}=A_{c c} V_{c c}=(b-a) a V
$$

Intake discharge 


$$
Q=Q_{c s}+Q_{c c}=A_{c s} V_{c s}+A_{c c} V_{c c}=V A=V a b
$$

in which $A=a b, A_{c s}=A_{c s \text { right }}+A_{c s \text { left }}, V_{c s}=$ average velocity at two CCHSSS(s), and $V=$ average velocity in the rectangular intake.

A rectangular intake may be located in many different positions. Four different cases (I, II, III and IV) that can frequently be met in practice are presented in figure 2 . In this figure, $l$ is the clearance of the dead-end wall to the intake center.

Yıldırım [15] and Yıldırım and Kocabaş [11] showed that if a single point sink and a single link sink are located in a uniform cross-flow separately, $V_{c s}$ and $V_{c c}$ become $U_{\infty} /$ 2 and $(2 / \pi) U_{\infty}$, respectively. $U_{\infty}$ is the average velocity of the incoming canal flow. Since it is very difficult to distinguish two half spherical sink flows and the velocities at the corresponding sink surfaces, Yıldırım [15] used Eq. (4) instead of Eqs. (1a, 1b). However, depending on geometrical and flow conditions, velocities at $\mathrm{CCHSSS}_{\text {left }}$ and CCHSSS $_{\text {right }}$; and CCCSS and CCHSSS(s) may be different from each other as well as the constant values that Yildirım [15] proposed $\left[V_{c c} \neq(2 / \pi) U_{\infty}\right.$ and $V_{c s}$ left $\neq V_{c s \text { right }} \neq$ $\left.U_{\infty} / 2\right]$. Therefore, critical submergences computed from Eqs. (1a-4) may differ from each other. Especially for rectangular intakes having large $b / a$, this difference may become significant depending on flow and geometrical conditions. Hence, Yıldırım [15] used Eq. (4) to obtain an average critical submergence for the whole intake.

In this study, because of the similar reasons stated earlier, Eq. (2) is used instead of Eqs. (1a, 1b), and $V_{c c}$ and $V_{c s}$ values are obtained from available experimental results for a rectangular intake by disregarding boundary blockage effects on sink surfaces $\left[A_{c s}=2\left(2 \pi S_{c}^{2}\right)\right.$ and $A_{c c}=2 \pi S_{c}$ $(b-a)]$. Therefore, $V_{c c}$ and $V_{c s}$ are different from those given by Yildirım [15], and Eqs. (2-4) give identical results. To determine the most convenient choice among Eqs. (2)-(4), it is necessary to examine the natures of $V_{c c}$ and $V_{c s}$. For this purpose, the available experimental test data of Yildırım [15] relating to an isolated rectangular intake located in an incoming cross-flow are plotted in figure 3 . This figure confirms that the relation between $Q_{c s}$ and $4 \pi S_{c}^{2}$ may be represented by a linear equation. In conjunction with Eq. (2), the values of $V_{c s}$ are equal to the slopes of the lines in figure 3(a). This means that $V_{c s}$ is a constant for given conditions. Figure $3 \mathrm{~b}$ indicates that the relation between $Q_{c c}$ and $2 \pi S_{c}(b-a)$ is nonlinear, which makes the prediction of $V_{c c}$ difficult. Whereas, since it is easy to predict $V_{c s}$, Eq. (2) should be preferred to Eqs. (3) and (4).

From Eq. (2),

$$
\frac{S_{c}}{a}=\frac{1}{2 \sqrt{\pi}}\left(\frac{V}{V_{c s}}\right)^{0.5}
$$

In conjunction with $V_{c s}$ values in figure 3a, Eq. (2) and the available test results relating to $S_{c} / a$ are compared in figure 4.
The method presented in Yildirım [15] overestimates the critical submergence especially for the cases where friction caused by the flow boundaries is effective on the development of the air-entraining vortex as indicated in figure 4. The reason of this is explained as follows. Y1ldırım [15] evaluated the velocities at critical hemispherical sink surfaces and critical cylindrical sink surface $\left(V_{c s}\right.$ and $V_{c c}$ ) by using potential flow theory in which boundary friction is not considered. However, the boundaries cutting or close to the free surface at the vicinity of the rectangular intake may introduce considerable friction effects that may retard the occurrence or development of air-entraining vortex [7]. Hence, the method presented in Yildırım [15] overestimates the critical submergence.

On the other hand, in this study, $V_{c s}$ and $V_{c c}$ are determined by using available experimental results and boundary friction effects are considered in the evaluation of $V_{c s}$ and $V_{c c}$. Therefore agreement between the available test data and theoretical results in this study is better than that of Yildırım [15].

It should be noted that $S_{c}$ computed from Eq. (2) is an average critical submergence for CCHSSS $_{\text {left }}$ and CCHSSS $_{\text {right }}$ whereas it is only a submergence $(S)$ for CCCSS. Similarly, $S_{c}$ computed from Eq. (3) is critical submergence for CCCSS whereas it is only an average submergence $(S)$ for $\mathrm{CCHSSS}_{\text {left }}$ and CCHSSS $\mathrm{Crght}_{\text {rig. }}$

If $Q_{c s}=0, S_{c} / a$ should be obtained from the following equation of continuity:

$$
Q=Q_{c c}=2 \pi S_{c} b V_{c c}=V a b \text { or } \frac{S_{c}}{a}=\frac{1}{2 \pi}\left(\frac{V}{V_{c c}}\right) .
$$

For given geometrical and flow conditions, $V_{c c}$ may be evaluated from Eq. (7), which is obtained from the ratio of discharge passing through CCHSSS(s) $\left(Q_{c s}\right)$ to discharge passing through CCCSS $\left(Q_{c c}\right)$.

$$
\frac{V_{c c}}{V_{c s}}=2\left(\frac{S_{c}}{a}\right) .
$$

\subsection{Scale effects}

Taştan and Yildırım [8] have proven that the ratio of the critical submergence to the diameter of the pipe intake is identical for the pipe intakes that have identical ratio of the intake velocity to the velocity at the critical spherical sink surface (CSSS), regardless of the flow and geometrical conditions and that only the kinematic similarity is required [Froude and Reynolds similarities (or dynamic similarity) are not necessary between the model and the prototype pipe intakes].

It is reasonable to think that this principle should also be applicable for a rectangular intake. Equation (5) infers that the rectangular intakes that have identical $V / V_{c s}$ have the same $S_{c} / a$ regardless of the flow and geometrical conditions. To obtain identical $V / V_{c s}$ in both the model and the 
Plan

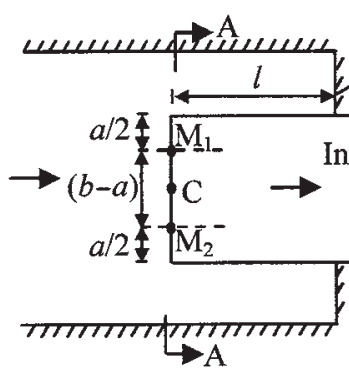

Plan

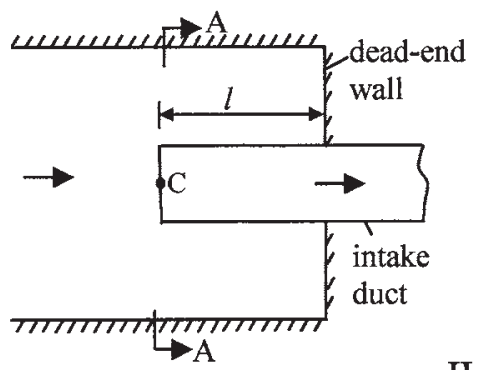

case II

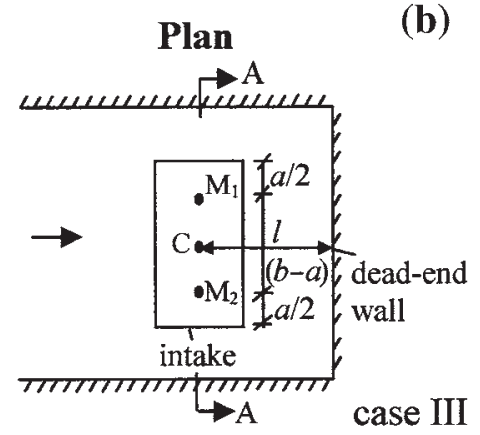

(c)

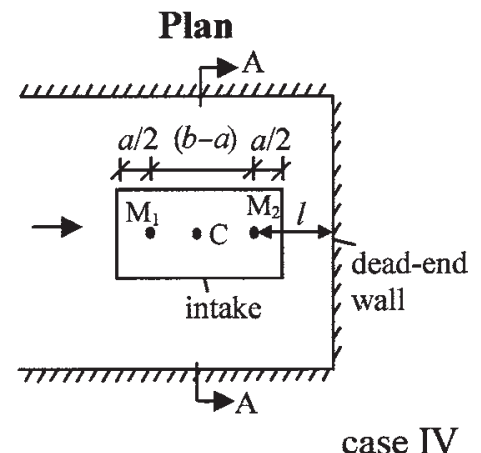

(d)
Section A-A

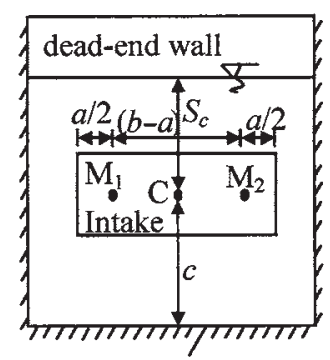

canal bottom

(a)

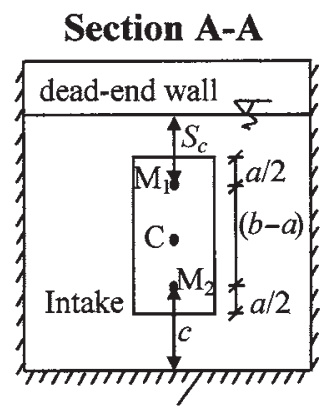

canal bottom

Section A-A

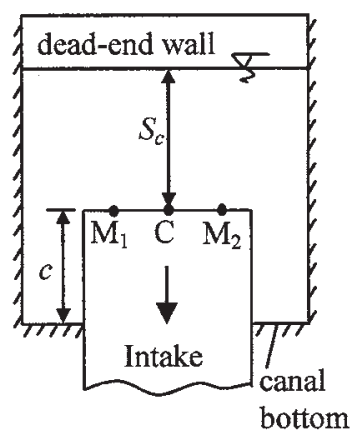

Section A-A

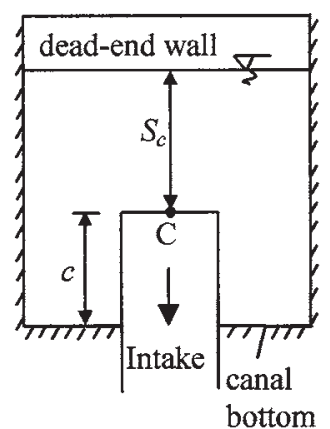

Figure 2. Various positions of a rectangular intake located in a canal flow with a dead-end wall: (a) Case I (long side horizontal rectangular intake horizontally passing through the dead- end wall), (b) Case II (long side vertical rectangular intake horizontally passing through the dead-end wall), (c) Case III (long side horizontal rectangular intake vertically flowing and passing through the canal bottom; its long side is normal to the canal flow), (d) Case IV (long side horizontal rectangular intake vertically flowing and passing through the canal bottom; its short side is normal to the canal flow). 
(a)

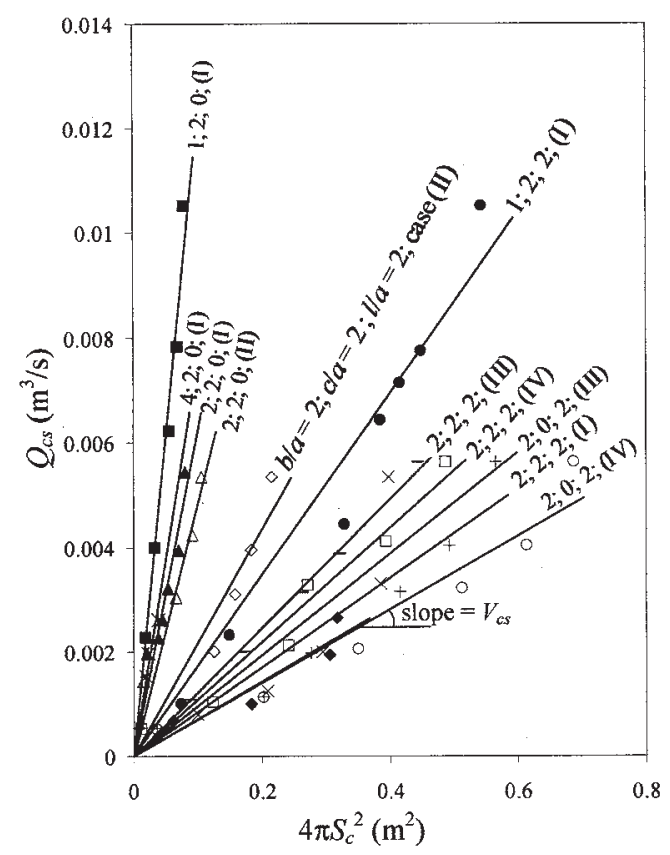

(b)

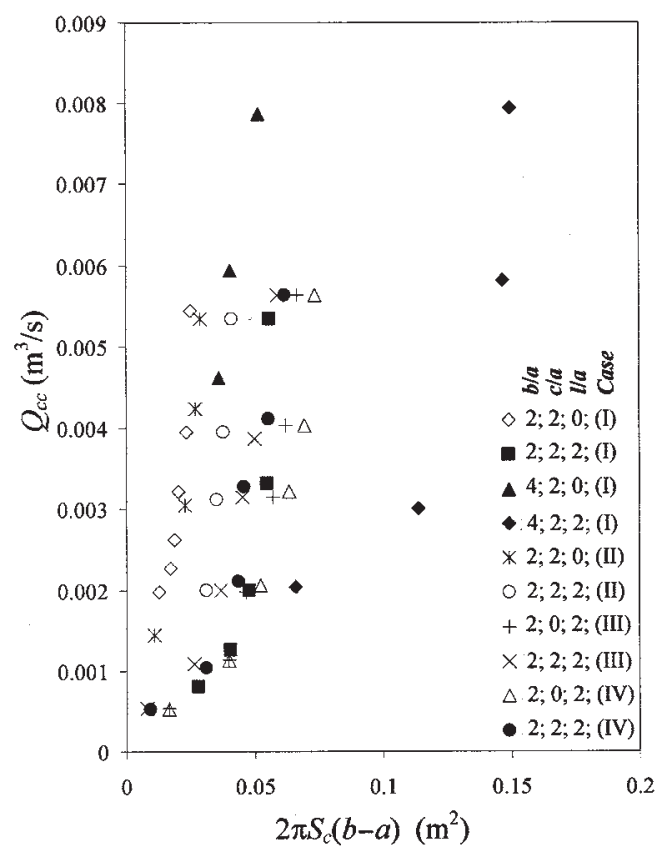

Figure 3. Relations between: (a) $Q_{c s}$ and $4 \pi S_{c}^{2}$, (b) $Q_{c c}$ and $2 \pi S_{c}(b-a)$ (test results are from [15]).

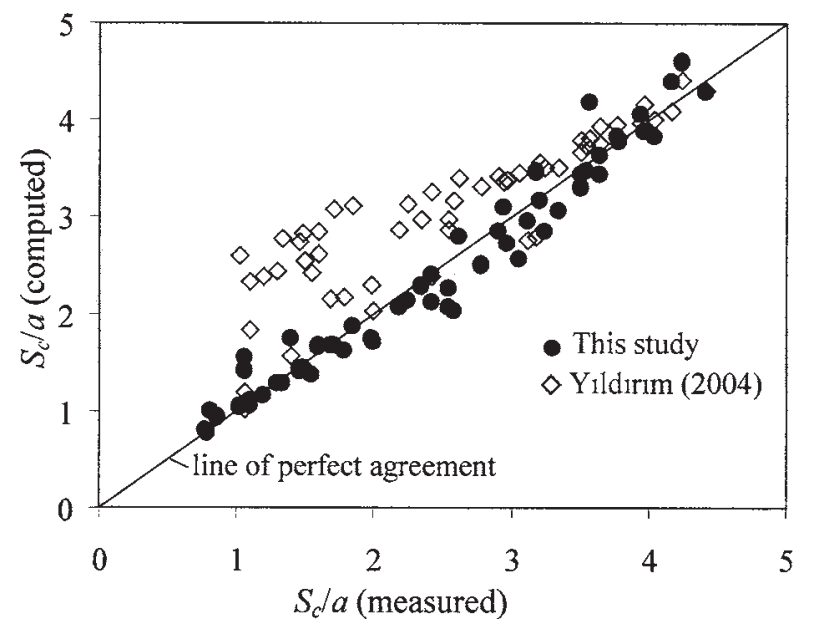

Figure 4. Comparison of theoretical and test results for an isolated rectangular intake.

prototype, practical methods similar to those explained in the study by Taştan and Yıldırım [8] may be utilized.

The validity of the kinematic similarity relating to the rectangular intakes of different sizes located in different conditions is examined. The available test data of Yildirım [15] are used and the results are presented in table 1. Equation (5) and the test results in table 1 confirm that the rectangular intakes that have identical $V / V_{c s}$ also have identical $S_{C} / a$ regardless of the conditions that the rectangular intakes are located in.
Table 1. Scale effects for an isolated rectangular intake.

\begin{tabular}{|c|c|c|c|c|c|c|c|}
\hline \multicolumn{5}{|c|}{ Experiment $^{\mathrm{a}}$} & \multicolumn{3}{|c|}{ Computed } \\
\hline$b / a$ & $c / a$ & $l / a$ & $Q(\mathrm{lt} / \mathrm{s})$ & $S_{c} / a$ & $V(\mathrm{~m} / \mathrm{s})$ & $V_{c s}(\mathrm{~m} / \mathrm{s})$ & $V / V_{c s}$ \\
\hline 2 & 2 & 2 & 7.90 & 2.42 & 1.58 & 0.02147 & 73.6 \\
\hline 4 & 2 & 2 & 4.00 & 2.42 & 0.40 & 0.00543 & 73.6 \\
\hline 2 & 2 & 0 & 4.54 & 1.10 & 0.908 & 0.05970 & 15.2 \\
\hline 4 & 2 & 0 & 10.5 & 1.10 & 1.050 & 0.06905 & 15.2 \\
\hline
\end{tabular}

Note that the rectangular intakes admitting flows only from their long sides and having identical $V / V_{c c}$ have the same $S_{c} / a$ as indicated by Eq. (6).

\section{Case of dual rectangular intakes}

Dual rectangular intakes may be used in practice to meet the discharge requirements. It is known that multiple intakes may introduce flow blockages [18-20]. Flow blockage represents an additional outflow for the sink surfaces besides the intake flow. Ylldırım et al [16] showed that critical submergence of a rectangular intake in dual intakes may be determined by the superposition method. The superposition method for dual rectangular intakes proposed by Yildirım et al [16] is adopted for this study by disregarding whole boundary blockages caused from the ducts of the intakes and the impervious flow boundaries. 


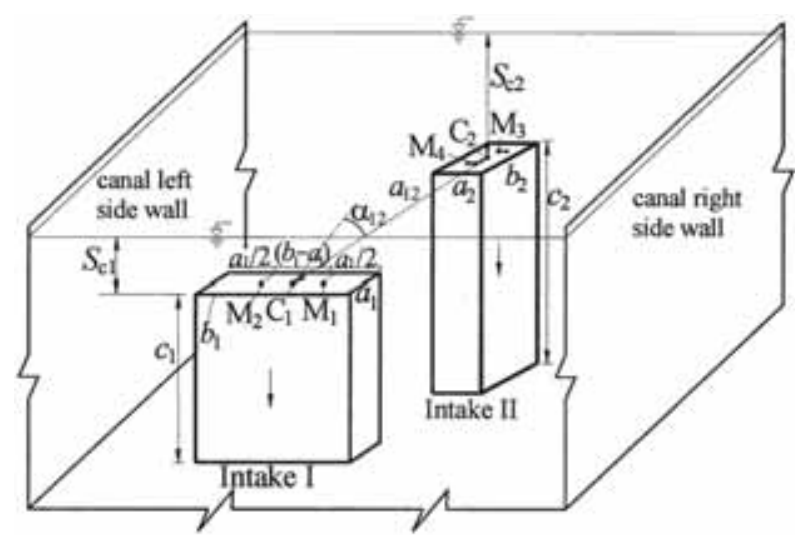

Figure 5. Parameters for dual rectangular intakes [16].
Thus, imaginary complete critical sink surfaces are used in the calculation of critical submergence.

Parameters describing the position of dual rectangular intakes are presented in figure 5. In figure 5; indices 1 and 2 represent intakes I and II, respectively; $a_{12}$ is the distance between $\mathrm{C}_{1}$ and $\mathrm{C}_{2}$, and $\alpha_{12}$ is the angle of the direction of incoming flow with the line between $\mathrm{C}_{1}$ and $\mathrm{C}_{2}$.

Herein, for a simple explanation, a case in which the CCHSSS(s) of an intake intersects the CHSSS(s) of the other intake (figure 6), and the CCCSS of an intake intersects the CCSS of the other intake (figure 7) is considered. Similar analysis may be done for other situations in which intersections of CCSS and CHSSS(s) are involved. CHSSS I denote two complete hemi-spherical sink surfaces of intake I, and CHSSS II denote two complete hemi-spherical

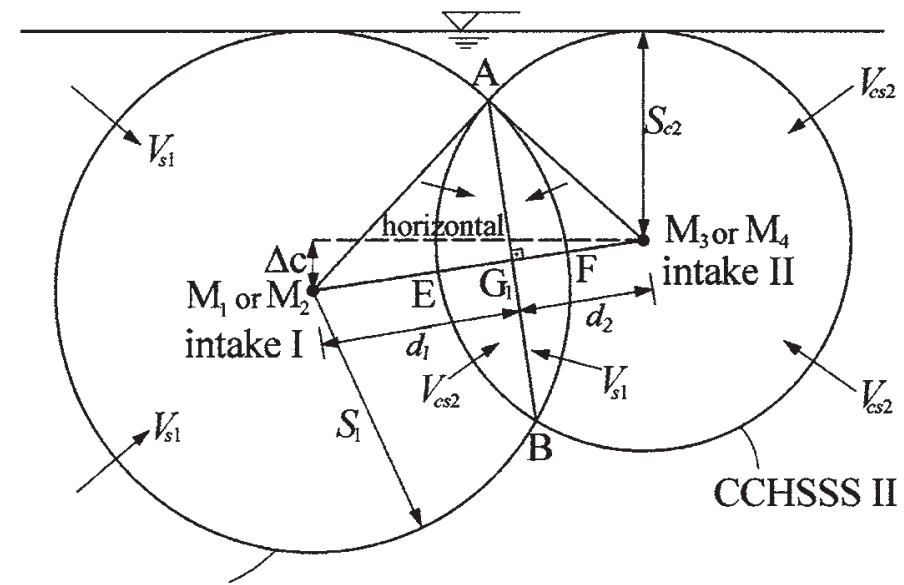

CHSSS I

Figure 6. Flow blockages due to intersection of the CHSSS(s) for the intakes I and II.

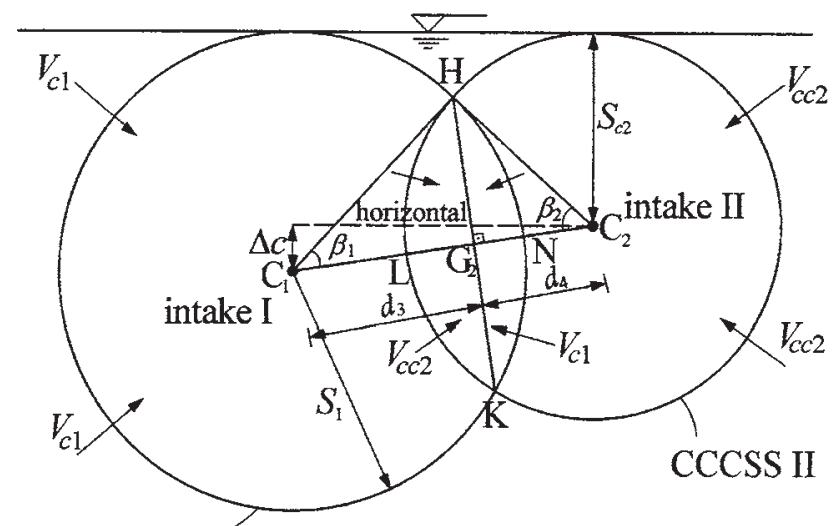

CCSS I

The lengths of CCSS(s) normal to the picture plane are $(b-a)$

Figure 7. Flow blockages due to intersection of the CCSS(s) for the intakes I and II. 


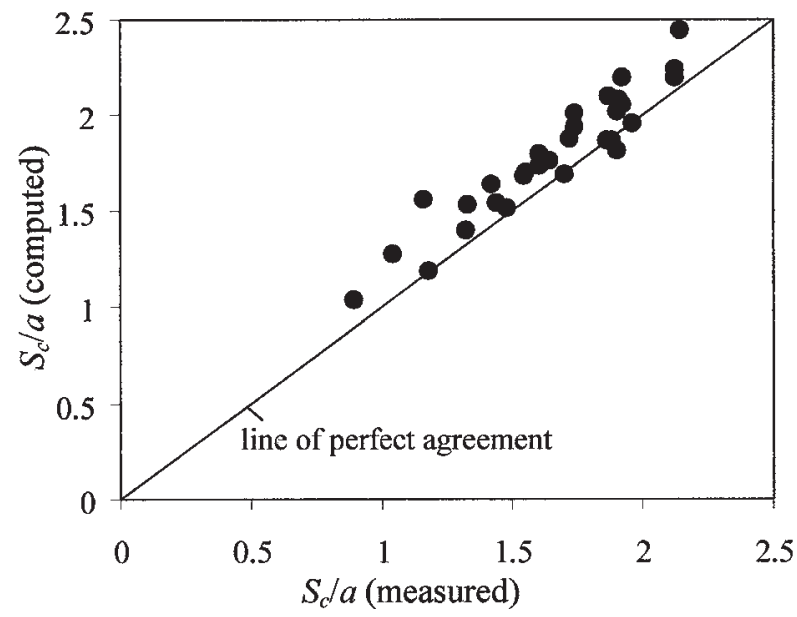

Figure 8. Comparison of theoretical and test results for dual rectangular intakes located at various positions.

sink surfaces of intake II. CCSS I and CCSS II refer to complete cylindrical sink surfaces for intakes I and II, respectively. The radii of CHSSS I and CCSS I, and CHSSS II and CCSS II are equal to submergences of intakes $\left(S_{1}\right.$ and $\left.S_{2}\right)$. For example, if critical case is considered for intake II, CHSSS II, CCSS II, and $S_{2}$ become CCHSSS II, CCCSS II and $S_{c 2}$, respectively. In figures 6 and 7; $V_{s 1}$ and $V_{c 1}$ are the velocities at CHSSS I and CCSS I, and $V_{c s 2}$ and $V_{c c 2}$ are the velocities at CCHSSS II and CCCSS II, respectively. Note that $S_{c 2}=S_{1}-\Delta c, \Delta c$ is the vertical difference between the centers of intake I and intake II.

The flow blockage effect of intake I on the intake II is equal to the discharge toward intake I through the complete surface of the spherical sector, (AFB), of CHSSS I remaining within CCHSSS II (figure 6) and the complete surface of the cylindrical sector, (HNK), of CCSS I remaining within CCCSS II (figure 7). Considering these explanations the continuity for the intakes can be written as follows (note that $Q_{1}$ is the discharge of intake I and $Q_{2}$ is the discharge of intake 2).

$$
\begin{aligned}
Q_{1}= & 2\left(2 \pi S_{1}^{2}\right) V_{s 1}-(\mathrm{AEB}) V_{c s 2}+\left[2 \pi S_{1}(b-a)\right] V_{c 1} \\
& -(\mathrm{HLK}) V_{c c 2} \\
Q_{2}= & 2\left(2 \pi S_{c 2}^{2}\right) V_{c s 2}-(\mathrm{AFB}) V_{s 1}+\left[2 \pi S_{c 2}(b-a)\right] V_{c c 2} \\
& -(\mathrm{HNK}) V_{c 1}
\end{aligned}
$$

$V_{c s 2}$ and $V_{c c 2}$ can be evaluated in the manner stated earlier by conducting experiments for an isolated intake II that has the identical conditions as in the group. However, there are still three unknowns $\left(V_{s 1}, V_{c 1}\right.$ and $\left.S_{c 2}\right)$ in Eq. (8a, 8b). Continuity of intake I for a complete sink surface dictates that,

$$
\frac{V_{c 1}}{V_{s 1}}=2\left(\frac{S_{1}}{a}\right)
$$

For given flow conditions, Eqs. (8a, 8b, 9) can be solved for $S_{c 2}$. To check the validity of the method proposed in this study, the available test results of Yildirım et al [16] are compared with Eqs. (8a, 8b, 9) in figure 8. An example solution for a typical case is given in "Appendix A".

\section{Conclusions}

Following conclusions are obtained from this study:

- The method introduced in this study may be utilized to find the critical submergence for isolated and dual rectangular intakes. Since this method does not require the determination of boundary blockages, it is easy to use in practice. The agreement between experimental and theoretical results is found to be good.

- In this method imaginary complete critical sink surfaces are considered and velocities at these sink surfaces are determined by conducting only few experiments. Since the velocities at sink surfaces are determined from experiments they describe the whole characteristics of the geometrical and flow conditions. Therefore this method may be applied for the intakes located in general geometrical and flow conditions (i.e., intake in still water, circulation imposed flow, non-developed cross-flow, multiple intakes, etc.).

Appendix A. Example solution for the case of dual rectangular intakes

Example solution of critical submergence for flowing vertically downward identical $\left(a_{1}=a_{2}=a, b_{1}=b_{2}=b\right)$ dual rectangular intakes is given (figure 9).

Dimensions of identical dual intakes are $a=0.05 \mathrm{~m}$ and $b=0.1 \mathrm{~m}$. The clearances of intakes to the bottom boundary are $c_{1} / a=2, c_{2} / a=3(\Delta c / a=1) . a_{12}=0.1118 \mathrm{~m}$ or $a_{12} /$ $a=2.236, \alpha_{12}=90^{\circ}, U_{\infty}=0.114 \mathrm{~m} / \mathrm{s}$, and intake discharges $Q_{1}=0.003 \mathrm{~m}^{3} / \mathrm{s}, Q_{2}=0.006 \mathrm{~m}^{3} / \mathrm{s}$. The computation of $S_{c 2} / a$ by the method proposed in this study is explained in the following. Note that, only the solution of the final trial is given here not to increase the length of the paper. $S_{c 2}$ is chosen as $S_{c 2}=0.102 \mathrm{~m}$ or $S_{c 2} / a=2.04 . \quad S_{1} / a=S_{c 2} /$ $a+\Delta c l a=3.04\left(S_{1}=0.152 \mathrm{~m}\right)$. From figures 6 and 7 ,

$$
\begin{aligned}
& \frac{d_{1}}{a}=\frac{d_{3}}{a}=\frac{1}{2} \frac{a_{n 12}}{a}+\frac{\frac{\Delta c}{a}\left(2 \frac{S_{1}}{a}-\frac{\Delta c}{a}\right)}{2 a_{n 12} / a} ; \\
& \frac{d_{2}}{a}=\frac{d_{4}}{a}=\frac{1}{2} \frac{a_{n 12}}{a}-\frac{\frac{\Delta c}{a}\left(2 \frac{S_{1}}{a}-\frac{\Delta c}{a}\right)}{2 a_{n 12} / a}
\end{aligned}
$$

in which, $a_{n 12}=a_{12}\left|\sin \alpha_{12}\right|$ ( $a_{n 12}$ is the distance between the projected center points of intakes I and II on the same plane which is perpendicular to incoming cross-flow)

$$
a_{n 12}=0.1118\left|\sin 90^{\circ}\right|=0.1118 \mathrm{~m}
$$

$\mathrm{HLK}=2 \beta_{2} S_{c 2}(b-a)$ and $\mathrm{HNK}=2 \beta_{1} S_{1}(b-a)$ (note that $\beta_{1}$ and $\beta_{2}$ are in radians) 


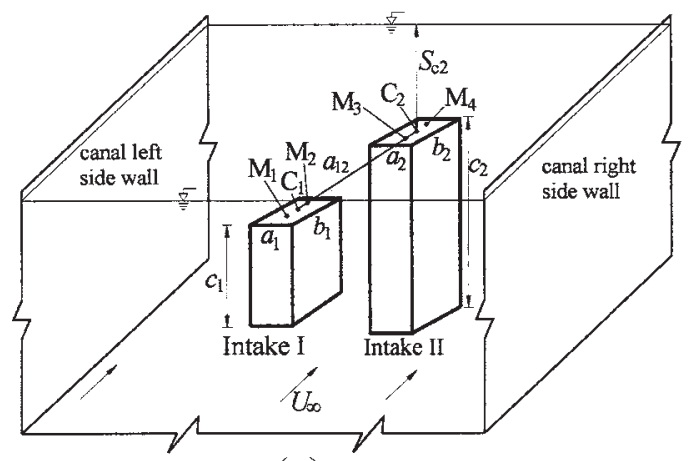

(a)

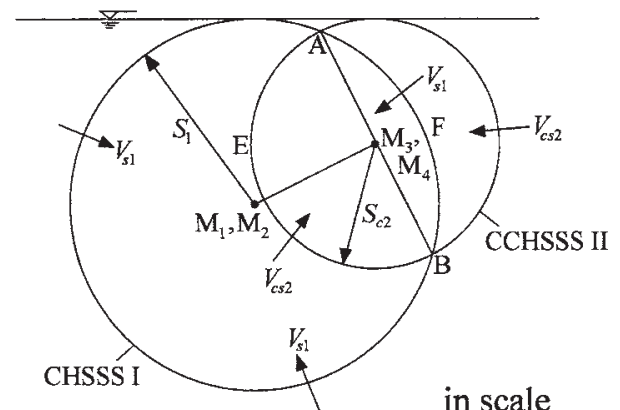

(b)

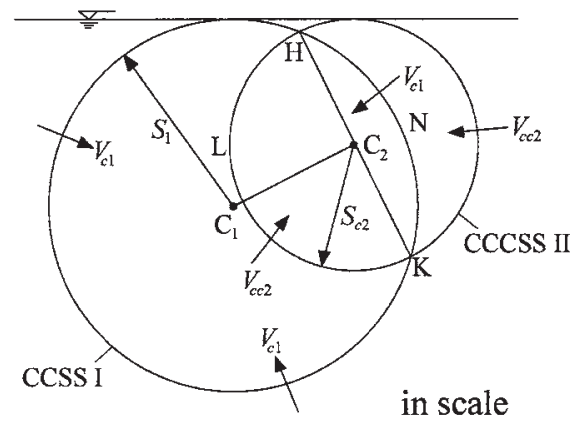

(c)

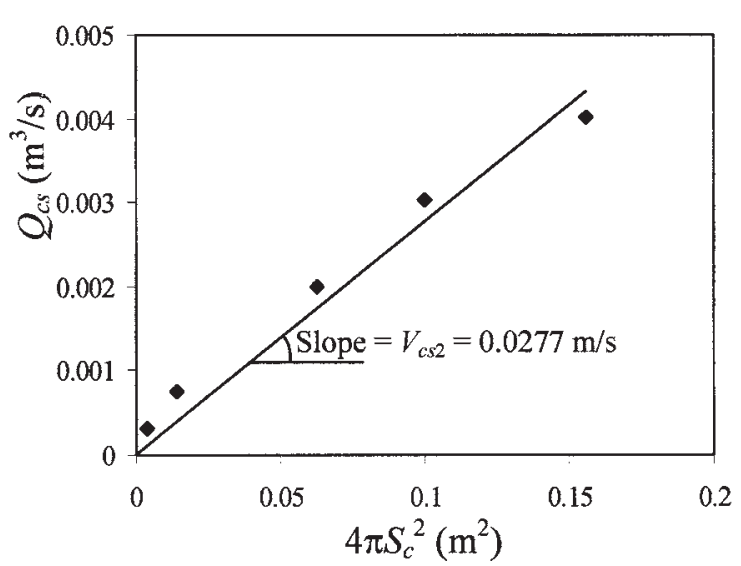

(d)

Figure 9. (a) Positions of the intakes in the channel, (b) flow blockages due to intersection of the CHSSS(s) for the intakes I and II for the example solution, (c) flow blockages due to intersection of the CCSS(s) for the intakes I and II for the example solution and (d) relation between $Q_{c s}$ and $4 \pi S_{c}^{2}$ for the isolated intake II.

$$
\begin{gathered}
\mathrm{AFB}=2 \pi S_{1}\left(S_{1}-d_{1}\right) \text { and } \mathrm{AEB}=2 \pi S_{c 2}\left(S_{c 2}-d_{2}\right) \\
d_{1}=d_{3}=\frac{0.1118}{2}+\frac{0.05(2 \times 0.152-0.05)}{2 \times 0.1118}=0.1127 \mathrm{~m} \\
d_{2}=d_{4}=\frac{0.1118}{2}-\frac{0.05(2 \times 0.152-0.05)}{2 \times 0.1118}=-0.0009 \mathrm{~m}
\end{gathered}
$$

Note that $d_{2}$ or $d_{4}$ can take a negative value when $\mathrm{G}_{1}$ or $\mathrm{G}_{2}$ is at the right hand side of $\mathrm{M}_{3}$ (figures 6 and 7).

In figure 9(b and c) CHSSS(s) and CCSS related to final solution of the example are drawn in scale. In figure $9(\mathrm{~b}$ and c);

$$
\begin{aligned}
& \mathrm{AFB}=2 \pi S_{1}\left(S_{1}-d_{1}\right)=2 \pi \times 0.152(0.152-0.1127) \\
& =0.0375 \mathrm{~m}^{2} \\
& \mathrm{AEB}=2 \pi S_{c 2}\left(S_{c 2}-d_{2}\right)=2 \pi \times 0.102(0.102-(-0.0009)) \\
& =0.0659 \mathrm{~m}^{2} \\
& \beta_{1}=\arccos \left(\frac{d_{3}}{S_{1}}\right)=\arccos \left(\frac{0.1127}{0.152}\right)=0.735 \\
& \beta_{2}=\arccos \left(\frac{d_{4}}{S_{c 2}}\right)=\arccos \left(\frac{-0.0009}{0.102}\right)=1.578 \\
& (\mathrm{HNK})=2 \beta_{1} S_{1}(b-a) \\
& =2 \times 0.735 \times 0.152 \times(0.1-0.05)=0.0112 \mathrm{~m}^{2} \\
& (\mathrm{HLK})=2 \beta_{2} S_{c 2}(b-a) \\
& =2 \times 1.578 \times 0.102 \times(0.1-0.05)=0.0161 \mathrm{~m}^{2}
\end{aligned}
$$

In critical condition, $V_{c s 2}$ for the isolated intake II is obtained from the available test results of Ylldirım et al [16]. In figure $9 \mathrm{~d}$, the slope of the straight line is equal to $V_{c s 2}=0.0277 \mathrm{~m} / \mathrm{s}$.

From Eq. (7)

$$
V_{c c 2}=2\left(\frac{0.102}{0.05}\right) \times 0.0277=0.113 \mathrm{~m} / \mathrm{s} .
$$

Considering the continuity equation of intake I by means of Eq. (8a)

$$
\begin{aligned}
0.003= & \left(4 \pi \times 0.152^{2} \times V_{s 1}-0.0659 \times 0.0277\right) \\
& +\left(2 \pi \times 0.152 \times 0.05 \times V_{c 1}-0.0161 \times 0.113\right) .
\end{aligned}
$$

From Eq. (9)

$$
\frac{V_{c 1}}{V_{s 1}}=2\left(\frac{0.152}{0.05}\right)=6.08
$$

From the two equations above $V_{s 1}$ and $V_{c 1}$ are calculated.

$$
V_{s 1}=0.0114 \mathrm{~m} / \mathrm{s} ; \quad V_{c 1}=0.0695 \mathrm{~m} / \mathrm{s}
$$

Check Eq. (8b) relating to intake II, 


$$
\begin{aligned}
0.006 \stackrel{?}{=} & \left(4 \pi \times 0.102^{2} \times 0.0277-0.0375 \times 0.0114\right) \\
& +(2 \pi \times 0.102 \times 0.05 \times 0.113-0.0112 \times 0.0695)
\end{aligned}
$$

$0.006 \cong 0.00603$ (therefore; further trial is not needed. The experimental value of critical submergence for intake II is $\left.S_{c 2} / a=1.90\right)$.

\section{Nomenclature}

A cross-section area of the intake

$A_{c c} \quad$ area of the CCCSS

$A_{c s} \quad$ total area of the CCHSSS(s)

$a$ inner dimension of the short edge of the rectangular intake

$a_{12}$ distance between $\mathrm{C}_{1}$ and $\mathrm{C}_{2}$

$b \quad$ inner dimension of the long edge of the rectangular intake

c clearance of the bottom boundary to the center of the intake level

$l$ distance between center point of the intake to the dead-end wall

$Q \quad$ intake discharge

$Q_{c c} \quad$ total discharge through CCCSS

$Q_{c s}$ total discharge through CCHSSS(s)

$S$ submergence

$S_{c} \quad$ critical submergence

$U_{\infty} \quad$ average velocity of cross-flow

$V \quad$ average intake velocity

$V_{c} \quad$ velocity at CCSS

$V_{c c} \quad$ velocity at CCCSS

$V_{c s}$ velocity at CCHSSS(s)

$V_{s} \quad$ velocity at CHSSS

$\alpha_{12}$ angle of the line connecting $\mathrm{C}_{1}$ and $\mathrm{C}_{2}$ with the direction of the incoming canal flow

\section{Subscripts}

$\begin{array}{ll}\text { left } & \text { for } \text { CCHSSS }_{\text {left }} \\ \text { right } & \text { for CCHSSS } \\ 1 & \text { Intake I } \\ 2 & \text { Intake II }\end{array}$

\section{References}

[1] Borghei S M and Kabiri-Samani A R 2010 Effect of antivortex plates on critical submergence at a vertical intake on critical submergence of an intake pipe. Int. J. Sci. Technol. Scientia-Iranica 17(2): 89-95
[2] Naderi V, Farsadizadeh D, Dalir A H and Arvanaghi H 2014 Effect of using vertical plates on vertical intake on discharge coefficient. Arabian J. Sci. Eng. 39(12): 8627-8633

[3] Trivellato F 2010 Anti vortex devices: Laser measurements of flow and functioning. Opt. Laser Eng. 48(5): 589-599

[4] Mahyari M N, Karimi H, Naseh H and Mirshams M 2010 Numerical and experimental investigation of vortex breaker effectiveness on the improvement in launch vehicle ballistic parameters. J. Mech. Sci. Technol. 24(10): 1997-2006

[5] Wang Y K, Jiang C B and Liang D F 2011 Study on the critical submergence of surface vortices and the design of anti-vortex intakes. Sci. China Tech. Sci. 54(4): 799-805

[6] Suerich-Gulick F, Gaskin S, Villeneuve M and Parkinson E 2014 Characteristics of free-surface vortices at low-head hydropower intakes. J. Hydraulic Eng. 140(3): 291-299

[7] Taştan K and Yıldırım N 2010 Effects of dimensionless parameters on air-entraining vortices. J. Hydraulic Res. 48(1): 57-64

[8] Taştan K and Yıldırım N 2014 Effects of Froude, Reynolds and Weber numbers on an air-entraining vortex. J. Hydraulic Res. 52(3): 421-425

[9] Sarkardeh H, Zarrati A R and Roshan R 2010 Effect of the intake head wall and trash rack on vortices. J. Hydraulic Res. 48(1): 108-112

[10] Yang J, Liu T, Bottacin-Busolin A and Lin C 2014 Effect of intake-entrance profiles on free-surface vortices. J. Hydraulic Res. 52(4): 523-531

[11] Yıldırım N and Kocabaş F 1995 Critical submergence for intakes in open channel flow. J. Hydraulic Eng. 121(12): 900-905

[12] Carriveau R, Kopp G and Baddour R 2009 Free-surface stretching-sustained intake vortices. J. Hydraulic Res. 47(4): 486-491

[13] Jiming M, Yuanbo L and Jitang H 2000 Minimum submergence before double-entrance pressure intakes. J. Hydraulic Eng. 126(8): 628-631

[14] Odgaard A J 1986 Free surface air core vortex. J. Hydraulic Eng. 112(7): 610-620

[15] Yildirım N 2004 Critical submergence for a rectangular intake. J. Eng. Mech. 130(10): 1195-1210

[16] Yıldırım N, Eyüpoğlu A S and Taştan K 2012 Critical submergence for dual rectangular intakes. J. Energy Eng. 138(4): 237-245

[17] Eroğlu N and Bahadırlı T 2007 Prediction of critical submergence for a rectangular intake. J. Energy Eng. 33(2): 91-103

[18] Yıldırım N, Akay H and Taştan K 2011 Critical submergence for multiple pipe intakes by the potential flow solution. J. Hydraulic Res. 49(1): 117-121

[19] Yildırım N, Taştan K and Arslan M M 2009 Critical submergence for dual pipe intakes. J. Hydraulic Res. 47(2): 242-249

[20] Yildırım N and Taştan K 2009 Critical submergence for multiple pipe intakes. J. Hydraulic Eng. 135(12): 1052-1062 